\title{
On the Efficient Broadcasting of Heterogeneous Services over Band-Limited Channels: Unequal Power Allocation for Wavelet Packet Division Multiplexing
}

\author{
Maurizio Murroni \\ CNIT-MCLab, Department of Electrical and Electronic Engineering, University of Cagliari, Piazza d'Armi, 09124 Cagliari, Italy \\ Correspondence should be addressed to Maurizio Murroni, murroni@diee.unica.it
}

Received 5 September 2007; Revised 2 November 2007; Accepted 4 December 2007

Recommended by Massimiliano Laddomada

Multiple transmission of heterogeneous services is a central aspect of broadcasting technology. Often, in this framework, the design of efficient communication systems is complicated by stringent bandwidth constraint. In wavelet packet division multiplexing (WPDM), the message signals are waveform coded onto wavelet packet basis functions. The overlapping nature of such waveforms in both time and frequency allows improving the performance over the commonly used FDM and TDM schemes, while their orthogonality properties permit to extract the message signals by a simple correlator receiver. Furthermore, the scalable structure of WPDM makes it suitable for broadcasting heterogeneous services. This work investigates unequal error protection (UEP) of data which exhibit different sensitivities to channel errors to improve the performance of WPDM for transmission over bandlimited channels. To cope with bandwidth constraint, an appropriate distribution of power among waveforms is proposed which is driven by the channel error sensitivities of the carried message signals in case of Gaussian noise. We address this problem by means of the genetic algorithms (GAs), which allow flexible suboptimal solution with reduced complexity. The mean square error (MSE) between the original and the decoded message, $\psi$ which has a strong correlation with subjective perception, is used as an optimization criterion.

Copyright () 2008 Maurizio Murroni. This is an open access article distributed under the Creative Commons Attribution License, which permits unrestricted use, distribution, and reproduction in any medium, provided the original work is properly cited.

\section{INTRODUCTION}

Unequal error protection (UEP) is a channel coding technique used to increase the robustness of data that exhibit different sensitivities to transmissionerrors. This is often the case of digital multimedia compressed streams such as JPEG2000 [1] or MPEG [2]. Due to the extensive use of predictive and variable length codes, a compressed stream is in general more vulnerable to data losses and transmission errors, which can desynchronize the decoder causing spatial and temporal error propagation [3]. In broadcasting, feedback channel is not available, thus UEP relies on differentiated forward error correction (FEC) coding [4]: depending on their sensitivities to channel errors, data are protected with codes with higher or lower error correcting capabilities. Reed-Solomon (RS) or Turbo Codes (TC) are frequently used $[5,6]$, but also more performing techniques, based on Rate-Compatible (RC) codes [7], have been proposed by the research community. Unequal power allocation (UPA) is an alternative UEP technique which is deployed when, for several reasons, FEC coding is not efficient [8]. For broadcasting multiplexed communications (e.g., DVB, DAB), for instance, the available channel bandwidth per service is a key constraint and the use of FEC-based UEP schemes is barely suitable. In fact, FEC is a discrete nature coding scheme. It is subjected to some constraint which restricts the protection level (i.e., the code rate) only to a set of fixed values. Therefore, the overhead introduced by FEC codes can be a significant limitation for the efficient use of the bandwidth. On the other hand, UPA aims at distributing the available budget power over the parts of the stream, according to their sensitivities to channel error, to achieve improved final quality on transmitted data without any increase of the transmission bandwidth. Basically, UPA is performed by assigning different power weights to the data according to their "importance" (i.e., channel error sensitivities) within the stream: higher transmission power is assigned to more sensible data. As to this, UPA is a "continuous" process in the sense that 
weights are chosen in a real set with an accuracy which can be a priori selected and in theory infinite. Therefore, against FEC, UPA allows more flexibility in the protection of sensible data.

Wavelet packet modulation for orthogonally multiplexed communication was introduced as a promising technique to improve performance of conventional FDM and TDM schemes in both Gaussian and impulsive noises [9-11]. The properties of wavelet packets are exploited to embed data into waveforms which are mutually orthogonal both in time and frequency. Several studies conducted on this technology have shown that opportune design allows minimizing the energy of timing error interferences, which impair conventional TDM systems [10]. The overlapping bandpass nature of the transmission pulses (i.e., wavelets) allows better exploitation of the bandwidth respect to classical FDM [10], and it also intrinsically mitigates fading effects [12]. Moreover, due to the scalability of its structure, wavelet packets permit to multiplex data with different format (e.g., JPEG2000 and MPEG2 ), therefore being a desirable choice for broadcasting heterogeneous services.

In this work, a UPA scheme for wavelet packet division multiplexing (WPDM) is proposed. UPA applied to WPDM consists on assigning different power to wavelet packets according to the importance of the message signals carried on. In other words, considering a generic bit pattern, individual bits are weighted differently taking the channel conditions (i.e., the signal-to-noise ratio (SNR)) into account and transmitted on separate wavelet packets. As to the optimization, we use the mean square error in the parameter domain

$$
\operatorname{MSE}_{u}=E\left\{[u(\tau)-\widehat{u}(\tau)]^{2}\right\}
$$

with $u(\tau)$ and $\hat{u}(\tau)$ being the transmitted and decoded parameter, respectively. The nontrivial complexity of the problem does not allow closed-form analytical solution, which, thus, has to be sought by numerical approach. In literature, solutions based on the gradient algorithm have been proposed [8]. The complexity of such optimization methods increases with the size (i.e., number of bits) of the frame to be transmitted. In this work, we address UPA by exploiting the potentialities of the Genetic Algorithm (GA) to reduce the computation complexity. The use of GA as to the weights optimization is one of the novel aspects of this work. A genetic algorithm [13] is a search technique used in computing to find true or approximate solutions to optimization and search problem. GAs are extensively used in literature in different application fields of communication engineering such as network design, unicast, and multicast routing [14-16]. They allow finding iterated numerical solution to complex problems with accuracy dependent on the number of iterations selected. The major advantage of genetic algorithms is their flexibility and robustness as a global search method. They can deal with highly nonlinear problems and nondifferentiable functions as well as functions with multiple local optima. They are also readily amenable to parallel implementation, which renders them appropriate in real-time adaptive communications, extensively used for reconfigurable broadcasting services.
Results show that the proposed UPA-WPDM scheme allows increasing resilience of data which exhibit different sensitivities to channel errors during their transmission over AWGN channel. The performance improvement in terms of quality achieved in the parameter domain (i.e., $\mathrm{MSE}_{u}$ ) has been proved against an equally distributed WPDM- and FEC-based UEP systems, in the presence of similar bandwidth constraint. Moreover, the bandwidth gain for target quality (i.e., fixed $\mathrm{MSE}_{u}$ ) at a fixed bit error rate has been evaluated beside UEP FEC-based techniques.

In the following section, an overview on the WPDM technology is given. Section 3 formally defines UPA for WPDM by describing in detail the weighting optimization procedure and the GA-based proposed solution. The performance of the proposed UPA-WPDM scheme on Gaussian channel is analyzed and compared to equally power distributed equivalent schemes and to channel coding UEP systems in Section 4. Conclusions follow in Section 5.

\section{WAVELET PACKET DIVISION MULTIPLEXING}

WPDM is a multiple signal transmission technique in which the message signals are waveform-coded onto wavelet packet basis functions for transmission. To define the wavelet packet basis functions, we refer to wavelet multiresolution analysis (MRA), the details of which can be found in a number of textbooks [17-23] and tutorial articles [24-31].

Let $g_{0}[n]$ be a unit-energy real causal FIR filter of length $N$ which is orthogonal to its even translates; that is, $\sum_{n} g_{0}[n] g_{0}[n-2 m]=\delta[m]$, where $\delta[m]$ is the Kronecker delta, and let $g_{1}[n]$ be the (conjugate) quadrature mirror filter $(\mathrm{QMF}), g_{1}[n]=(-1)^{n} g_{0}[N-1-n]$. If $g_{0}[n]$ satisfies some mild technical conditions $[17,31]$, we can use an iterative algorithm to find the function $\phi_{01}(t)=\sqrt{2} \sum_{n} g_{0}[n] \phi_{01}(2 t-$ $n T_{0}$ ) for an arbitrary interval $T_{0}$. Subsequently, we can define the family of functions $\phi_{l m}, l \geq 0,1 \leq m \leq 2^{l}$ in the following (binary) tree-structured manner:

$$
\begin{gathered}
\phi_{l+1,2 m-1}(t)=\sum_{n} g_{0}[n] \phi_{l m}\left(t-n T_{l}\right), \\
\phi_{l+1,2 m}(t)=\sum_{n} g_{1}[n] \phi_{l m}\left(t-n T_{l}\right),
\end{gathered}
$$

where $T_{l}=2^{l} T_{0}$. For any given tree structure, the function at the leafs of the tree forms a wavelet packet. They have a finite duration, $(N-1) T_{l}$, and are self- and mutually-orthogonal at integer multiples of dyadic intervals, and hence they are a natural choice for scalable multiplexing applications $[9,10]$. In Figure 1, the wavelet packet functions (a) and the relevant power spectrum (b) for three-level (i.e., eight size wavelet packet) standard 12-tap Daubechies filters decomposition [23].

In WPDM, binary messages $x_{l m}[n]$ have polar representation (i.e., $x_{\operatorname{lm}}[n]= \pm 1$ ), waveform-coded by pulse amplitude modulation (PAM) of $\phi_{l m}\left(t-n T_{l}\right)$ and then added together to form the composite signal $s(t)$. WPDM can be implemented using a transmultiplexer and a single modulator 


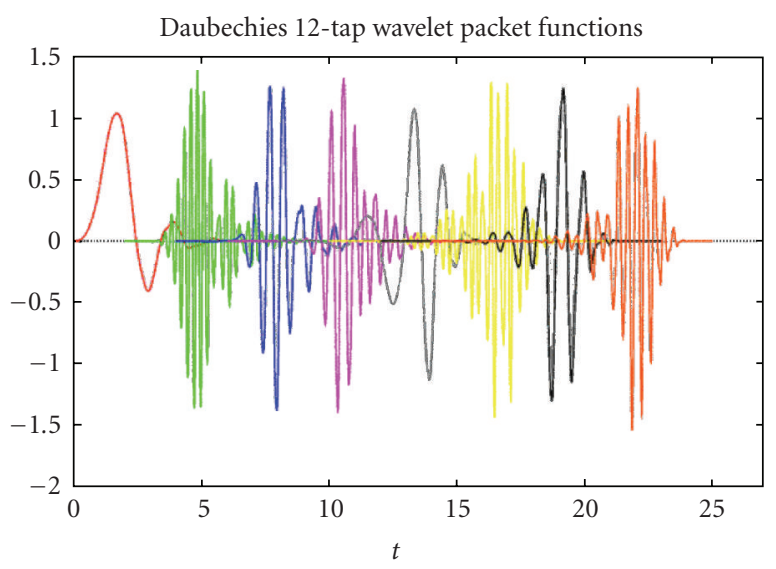

(a)

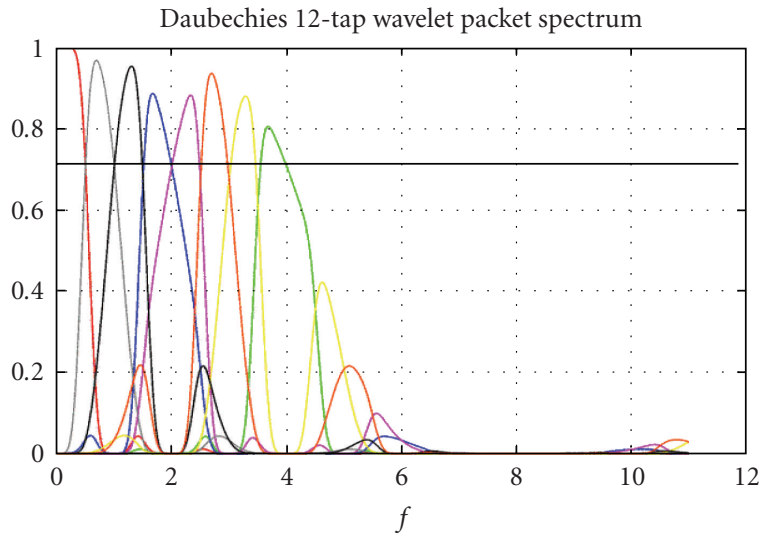

(b)

FIGURE 1: (a) Time and (b) frequency portrait for 12-tap Daubechies wavelet packets.

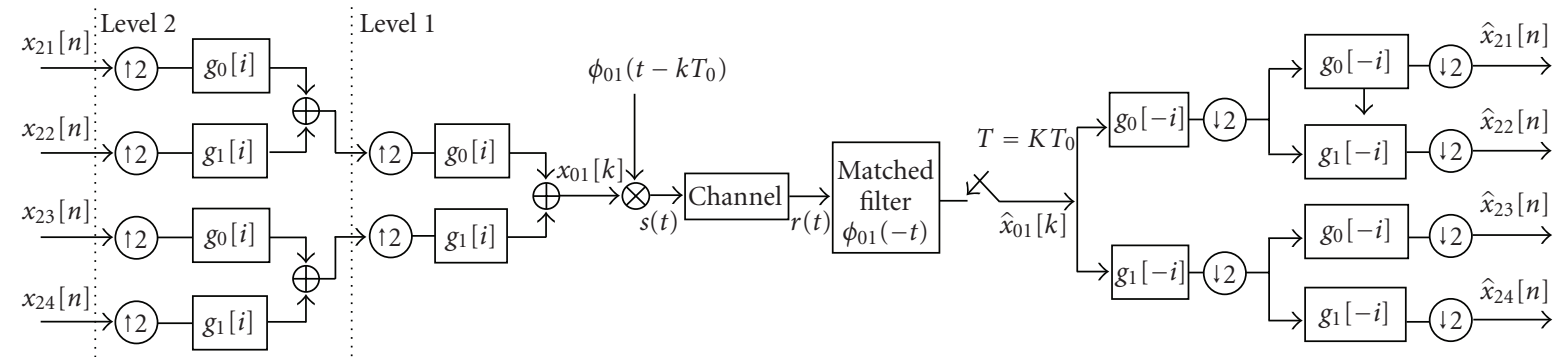

FIGURE 2: Transmitter and receiver for two-level WPDM system.

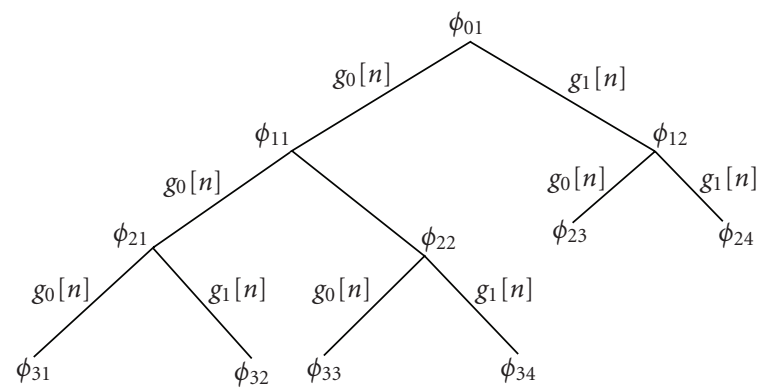

(a)

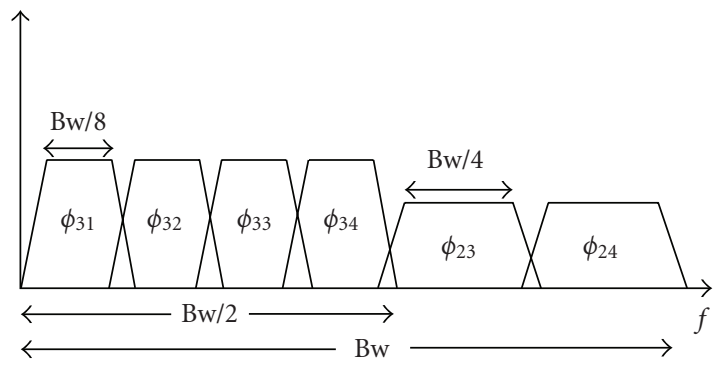

(b)

FIGURE 3: (a) WPDM tree structure suitable for broadcasting heterogeneous services. (b) Symbolic subband structure of the system in (a).

[10] as Figure 2 illustrates for a two-level decomposition. In this case,

$$
s(t)=\sum_{k} x_{01}[k] \phi_{01}\left(t-k T_{0}\right),
$$

where $x_{01}[k]=\sum_{(l, m) \in \Gamma} \sum_{n} f_{l m}\left[k-2^{l} n\right]$, with $\Gamma$ being the set of terminal index pairs and $f_{l m}[k]$ the equivalent sequence filter from the $(l, m)$ th terminal to the root of the tree, which can be found recursively from (2). The original message can be recovered from $x_{01}[k]$ using

$$
x_{l m}[n]=\sum_{k} f_{l m}\left[k-2^{l} n\right] x_{01}[k]
$$

An example of WPDM tree for a system that can be used for broadcasting heterogeneous services is shown in Figure 3(a). In this case, the transmission system uses two 
wavelet packets composed by two and four waveforms (i.e., wavelets), respectively. In Figure 3(b), the relevant subband structure is displayed: the total bandwidth is equally shared between the two packets, but a different partitioning (two against four) is implemented within each packet. Differently formatted streams can be transmitted by associating them to the appropriate wavelet packets.

\section{UNEQUAL POWER ALLOCATION FOR WPDM}

Without loss of generality to model, a generic bitstream exhibits different error sensitivities to channel conditions, we consider a discrete periodic (period $\tau$ ) memoryless source $S: \forall \tau \rightarrow u(\tau)$ and an analog to digital process $\mathrm{AD}$ : $\forall u(\tau) \rightarrow \underline{x}(\tau)$ with $\underline{x}(\tau) \in\left(\underline{x}_{k} \mid k=1,2, \ldots, 2^{M}\right), \underline{x}_{k}=$ $\left(x_{k}^{(1)}, x_{k}^{(2)}, \ldots, x_{k}^{(M)}\right), x_{k}^{(M)}$ being the LSB. Each $x_{k}^{(i)}$ is then multiplied with the specific weight $w_{i} \in \mathbb{R}^{+}$of the diagonal matrix $\underline{W}=\operatorname{diag}\left(w_{1}, w_{2}, \ldots, w_{M}\right)$. The weighted bit pattern $y(\overline{\tau)}=\underline{W} \cdot \underline{x}(\tau)$ is then transmitted by a $M$ th order WPDM over a channel affected by additive white Gaussian noise (AWGN) $n(t)$ with zero mean and variance $N_{0} / 2$. The signal at the receiver front end is $r(t)=s(t)+n(t)$ with $s(t)$ as in (1) and $T_{0}=2^{-l} \tau$.

After demodulation, the distributed vector is $\underline{z}(\tau)=$ $\underline{y}(\tau)+\underline{n}_{\text {rel }}(\tau)$, where $\underline{n}_{\text {rel }}=\left(n_{\text {rel }}^{(1)}, n_{\text {rel }}^{(2)}, \ldots, n_{\text {rel }}^{(M)}\right)$, represents the demodulated noise along the $M$ signal message components (i.e., relevant noise). Following decision based on Maximum Likelihood (ML) criterion, the estimate $\hat{u}(\tau)$ is produced by inverse digital to analog (DA) process. A sketch of the system is depicted in Figure 4.

\subsection{Weight optimization}

Considering bipolar binary representation $x_{k}^{(i)}= \pm 1$, if bits in $\underline{x}(\tau)$ are inverteddue to AWGN, a wrong decision $\hat{x}(\tau)$ is made at the receiver, thus producing a distortion $d(\tau)=$ $[u(\tau)-\hat{u}(\tau)]$. Aim of the optimization process is to calculate optimal weights in the sense of a minimized expected value $E\left\{\left[d^{2}(\tau)\right]\right\}$. Assuming ergodicity, it is possible to calculate $E\left\{d^{2}\right\}$ as follow:

$$
E\left\{d^{2}\right\}=\sum_{k=1}^{2^{M}} \sum_{h=1}^{2^{M}} d_{k, h}^{2} P\left(\underline{x}_{k}\right) \cdot P\left(\underline{\hat{x}}_{h} \mid \underline{x}_{k}\right),
$$

where $d_{\varsigma, \eta}=u_{\varsigma}-\hat{u}_{\eta}$ are the different possible parameter values, $P\left(\underline{x}_{k}\right)$ the occurrence of the reproduction levels $u_{k}$, and $P\left(\underline{\hat{x}}_{h} \mid \underline{x}_{k}\right)$ the transition probabilities between transmitted and received bit patterns. Due to the orthogonal properties of WPDM waveforms and to the independence of the noise samples, the transition probabilities are $[4,32]$ :

$$
P\left(\underline{\hat{x}}_{h} \mid \underline{x}_{k}\right)=\left(\prod_{\substack{i=1 \\ x_{k}^{(i)} \neq \hat{x}_{h}^{(i)}}}^{M} P_{b}^{(i)}\right) \cdot\left(\prod_{\substack{i=1 \\ x_{k}^{(i)}=\hat{x}_{h}^{(i)}}}^{M}\left(1-P_{b}^{(i)}\right)\right) .
$$

By imposing $E_{b}=(1 / M) \sum_{i=1}^{M} E_{b}^{(i)}=1$, we can write $[8] E_{b}^{(i)}=$ $w_{i}^{2} E_{b}$ and impose the following constraint on the weights $w_{i}$

$$
\sum_{i=1}^{M} w_{i}^{2}=M
$$

WPDM is based on binary amplitude modulation, thus, the bit error probabilities in (6) are [33]

$$
P_{b}^{(i)}=\frac{1}{2} \operatorname{erfc}\left(\frac{w_{i}}{\sqrt{N_{0}}}\right)
$$

Mathematically, the optimization problem is to minimize (5) under the constraint (7). In other words, UPA raises $\left(w_{i}>\right.$ 1) the immunity to noise channel for more significant bits, paying as a counterpart lower robustness $\left(w_{i}<1\right)$ on less significant one, to achieve average improved performance on the transmission of parameter $u(\tau)$ in the sense of minimum expected distortion $d(\tau)=[u(\tau)-\hat{u}(\tau)]$.

The complexity of the above optimization problem, which increases with the size of frames $M$, does not allow closed form solutions. Therefore, to identify the solution, we use a numerical approach based on Genetics Algorithms (GAs).

\subsection{Genetics alghoritms (GAs)}

GAs are implemented as a computer simulation in which a population of abstract representations (chromosomes) of candidate solutions (genes) to an optimization problem evolves toward better solutions. The evolution usually starts from a population of randomly generated chromosomes and happens in generations. In each generation, the fitness of every chromosome in the population is evaluated, multiple chromosomes are stochastically selected from the current population (based on their fitness), and modified (mutated or recombined) to form a new population. The new population is then used in the next iteration of the algorithm.

In the proposed system, the chromosomesare defined as arrays of $M$ genes $w_{i} \in \mathbb{R}^{+}$. The range of possible values of $w_{i}$ is constrained by (7). An initial population $\{$ INIT $\}$ of $L$ chromosomes is randomly selected. The fitness function is as defined as in (5). Two operations are allowed to determine the evolution of the initial population: crossover (with probability $\left.P_{\text {cross }}\right)$ used to interchange the elements of two chromosomes and mutation (with probability $P_{\text {mut }}$ ) which modify the value of one or more genes within a chromosome with the aim of leading the search out of local optima. In particular, the most fitting part of the population $\{$ BEST $\}$ is selected and directly inserted in the new generation, while the rest of the population $\{$ WORST is discarded and replaced by a subpopulation created by means of the crossover and mutation operators. In the case of two identical chromosomes resulted after the crossover and mutation operations, two individuals are randomly generated. The termination condition is satisfied once either the algorithmreaches a selected number of iterations (IT) or the fitness function maintains the same value for IT $_{\text {MAX }}$ iterations. At the end of the process, the chromosome with low score in the fitness function (i.e., 


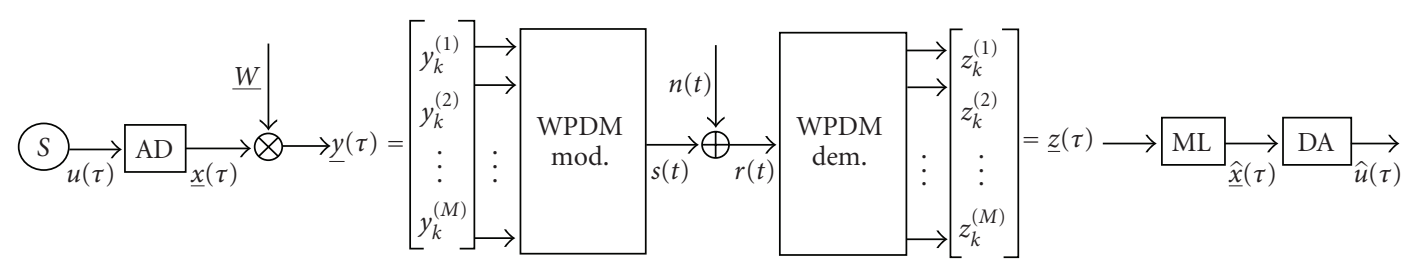

FIgURe 4: System model for UPA-WPDM.

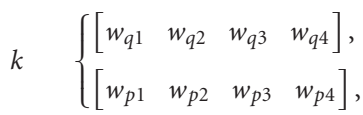

$$
\begin{aligned}
& \text { Crossover } k+1\left\{\begin{array}{llll}
{\left[w_{p 1}\right.} & w_{p 2} & w_{q 3} & w_{q 4}
\end{array}\right], \\
& \begin{array}{ll}
k & {\left[\begin{array}{llll}
w_{r 1} & w_{r 2} & w_{r 3} & w_{r 4}
\end{array}\right],} \\
\text { Mutation } & k+1\left[\begin{array}{lllll}
w_{r 1} & \left(w_{r 2} \cdot \Delta_{2}\right) & w_{r 3} & \left(w_{r 4} \cdot \Delta_{4}\right)
\end{array}\right] .
\end{array}
\end{aligned}
$$

FIgURE 5: Example for crossover and mutation operators in case of chromosomes composed by four genes.

lower distortion on the reconstructed frame) will be selected for the transmission.

Figure 5 gives an example of the crossover and mutation operations.

In this particular case, chromosomes are composed by four genes; at iteration $k+1$, the crossover operator swaps the first two genes of the chromosomes $p$ and $q$ as they were at iteration $k$, whereas the mutation varies the chromosome $r$ by multiplying the second and fourth genes for the quantity $\Delta_{i} \in \mathbb{R}^{+}$with $i=\{2,4\}$, respectively. The flowchart of the proposed GA is shown in Figure 6.

The accuracy of such approach is strictly dependent on the values of IT and IT $_{\mathrm{MAX}}$, whereas the complexity of the algorithm depends also on the definition of chromosomes, on the size $L$ of the initial population and on the $P_{\text {cross }}$ and $P_{\text {mut }}$ probabilities. Chromosomes are arrays of genes which are real values. The higher the precision on the representation of the genes (i.e., the number of decimal digits used to approximate real values), the higher the accuracy achieved by the UPA, but also, the higher the complexity of the algorithm. Similarly, big-size populations guarantee higher performance, but also lead to time consuming processing. A critical matter is the selection of $P_{\text {cross }}$ and $P_{\text {mut }}$ probabilities: high values can determine instability of the GA which could diverge, whereas, on the other side, low values likely lead to slow convergence.

\section{RESULTS}

A WPDM system which deploys two packets of size $M=$ $\{4,8\}$ is used to multiplex two streams having same rate, but different format (see Figure 7). Standard Daubechies minimum-phase scaling filters of length $N=12$ [31], which guarantee short delay and substantial capacity advantage over conventional FDM systems [10], are deployed. Without

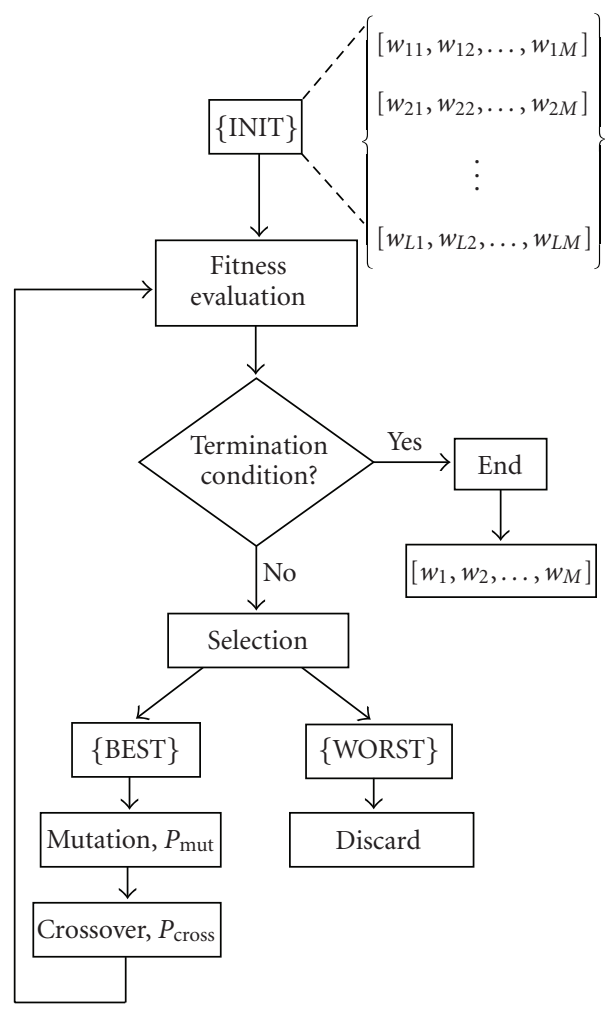

FIgURE 6: Flowchart of the proposed GA.

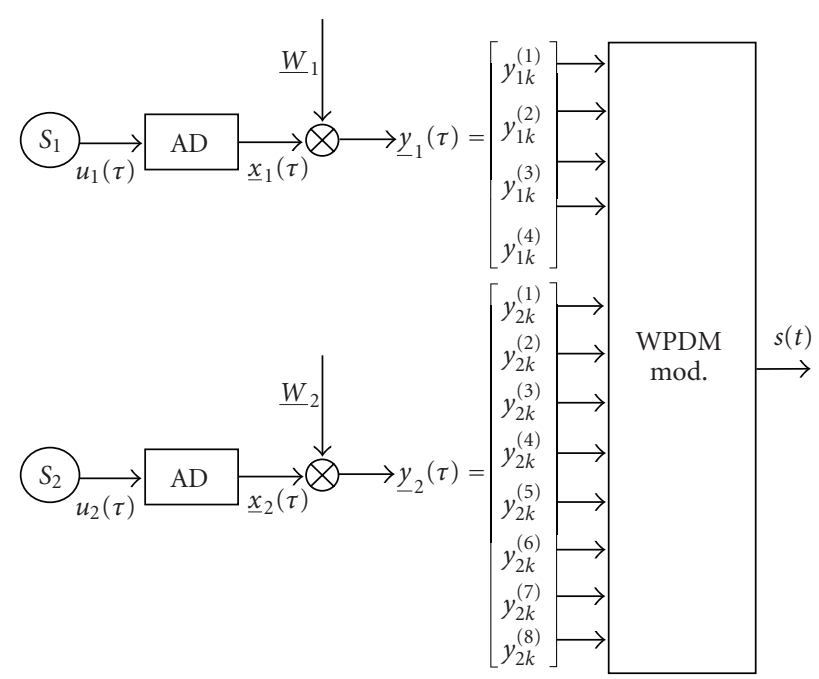

FIGURE 7: UPA-WPDM system for broadcasting two heterogeneous services. 
TABLE 1: Parameters setting for experiments.

\begin{tabular}{lll}
\hline Symbol & Definition & Setting \\
\hline$S_{1}, S_{2}$ & Source & Gaussian $\left(\eta=0, \sigma^{2}=1\right)$ \\
AD/DA & Analog to digital/digital to analog processes & Uniform quantizer, natural binary mapping \\
$M$ & Bit frame size (chromosome size) & 8 \\
$L$ & Size of initial population & 32 \\
$w_{i}$ & Weight (gene) & $\in \mathbb{R}^{+}, 8$ decimal digits precision \\
$P_{\text {mut }}$ & Mutation probability & 0.3 \\
$\Delta_{i}$ & mutation quantity & $0.25 \cdot w_{i}$ \\
$P_{\text {cross }}$ & Crossover probability & 0.5 \\
$I^{T}$ & Number of iteration & 1000 \\
IT $_{\text {MAX }}$ & Maximum number of iteration with unchanged fitness & 100 \\
\hline
\end{tabular}

loss of generality, to model the parameters $u_{1}(\tau)$ and $u_{2}(\tau)$ delivered at time $\tau$, we use zero-mean $(\eta=0)$ Gaussian sources $S_{1}$ and $S_{2}$ with unitary variance $\left(\sigma^{2}=1\right)$. AD/DA processes deploy natural binary mapping based on uniform quantizers.

At first, we have run some preliminary tests to analyze the importance of the GA parameters. The crossover operator was allowed to interchange int $[0.4 \cdot M]$ genes whereas the mutation occurred on int $[0.1 \cdot M]$ genes,int $[\cdot]$ being the operator which produces the integer part of the argument. In other words, at each iteration, a maximum of $40 \%$ of the chromosome parents could appear on the next generation of chromosomes and only $10 \%$ of a chromosome could vary. According to this, $L$ was varied in the range $\{8,16,32,64,128\}$, $P_{\text {cross }}$ and $P_{\text {mut }}$ in the range $0.3 \div 0.7$ and $0.01 \div 0.3$, respectively. Finally for mutation $\Delta_{i}$ varied within the range $\{0.1,0.2,0.3\} \cdot w_{i}$. The maximum difference in terms of fitness function value among all the solutions was observed to be less than 5\%. Therefore, the following considerations can be made: huge-size populations bring to better solutions at the expense of a higher-processing time; the $P_{\text {mut }}$ probability is suggested to be set equal to or higher than 0.1 , whereas $\Delta_{i}$ above $0.2 \cdot w_{i}$ to avoid an excessive number of iterations; the $P_{\text {cross }}$ probability does not sort significant effects in the range used. As to the outcome from the preliminary tests on GA behaviour applied to the UPA problem, in the following experiments the $\{$ INIT $\}$ population was composed by $L=32$ chromosomes, eight decimal digits were used to represent genes (i.e., $w_{i}$ ), the probability $P_{\text {mut }}=0.3,0.25 \cdot w_{i}$ and $P_{\text {cross }}=0.5$, whereas $\mathrm{IT}_{\mathrm{MAX}}=100$ and IT $=1000$. For the sake of clearness, Table 1 summarizes the parameter setting for the experiments.

Achieved quality in the parameters domain is expressed in terms of the signal-to-noise ratio $\left(\mathrm{SNR}_{u}\right)$ measured in decibel $\mathrm{SNR}_{u}[\mathrm{~dB}]=10 \cdot \log _{10}\left[E\left\{u^{2}(\tau)\right\} / \mathrm{MSE}_{u}\right]$ with $\mathrm{MSE}_{u}$ as in (1). $\mathrm{SNR}_{u}[\mathrm{~dB}]$ is evaluated at varying average bit error probabilities $P_{b}=(1 / M) \sum_{i=1}^{M} P_{b}^{(i)}$ with $P_{b}^{(i)}$ as in (8).

We have compared the proposed UPA with a benchmark equal power allocation (EPA) WPDM system and an UEP scheme based on FEC coding. In the latter system, we have deployed Reed-Solomon (RS) codes [33]. RS codes are nonbinary cyclic codes with symbols made up of $m$-bit sequences, where $m$ is any positive integer having a value greater than
TABLE 2: Actual parameters for Reed-Solomon UEP coding at $P_{b}=$ $10^{-3}$ for $u_{1}(\tau)$ transmission.

\begin{tabular}{lcc}
\hline RS $\left(n^{i}, k^{i}\right)$ & $R_{c}^{i}$ & $t^{i}$ \\
\hline RS $(38,24)$ & 0.63 & 7 \\
RS $(38,30)$ & 0.79 & 4 \\
RS $(38,34)$ & 0.89 & 2 \\
RS $(38,36)$ & 0.95 & 1 \\
\hline
\end{tabular}

2. RS $(n, k)$ codes on $m$-bit symbols exist for all $n$ and $k$ for which $0<k<n<2^{m}+2$, where $k$ is the number of data symbols being encoded, and $n$ is the total number of code symbols in the encoded block. The error-correcting capability of the generic RS $(n, k)$ code is $t=(n-k) / 2$. UEP is implemented by protecting data with codes with higher- or lowercode rate $R_{c}^{i}=k^{i} / n^{i}$. At varying the channel error rate, for every WPDM channel, an appropriate RS $\left(n^{i}, k^{i}\right)$ code is selected for data protection according to the sensitivity to channel errors of the data carried on. More significant data (e.g., MSB) are protected by codes with higher error-correcting capabilities (i.e., higher-code rates). In particular, for any average error rate $P_{b}$, the optimization procedure aims at selecting the $M$ codes so that the $\mathrm{SNR}_{u}$ is minimized under the bound of constant average code rate $R_{c}=(1 / M) \sum_{i=1}^{M} R_{c}^{i}$.

For our experiments, we have selected $m=8$ and $R_{c}=$ $32 / 38=0.84$ which corresponds to an increase of the total bandwidth of about $16 \%$. To reduce the complexity of the coding process, we have fixed the number of code symbols in the encoded block $n^{i}=38$. The average error correcting capability of the system is therefore $t=(38-32) / 2=3$ symbols per codeword. In other words, on the average, such a scheme is able to correct up to 3 symbols that contain errors in a codeword. Tables 2 and 3 report the details (i.e., actual code rate $R_{c}^{i}$ and error correcting capability $t^{i}$ ) of the codes used at $P_{b}=10^{-3}$ for the transmission of $u_{1}(\tau)$ and $u_{2}(\tau)$, respectively.

In Figures 8 and 9, we refer to UEP RS-based coding as RS $(38,32)$. The analysis of the graphics reveals that UPA outperforms EPA along all the variation ranges of the average bit error probability within the transmitted frame with a peak gain of $6.84 \mathrm{~dB}$ at $P_{b}=10^{-3}$ in case of $u_{2}(\tau)$. Same behaviour is noticeable with respect to RS coding for $P_{b}>10^{-4}$, with 
TABLe 3: Actual parameters for Reed-Solomon UEP coding at $P_{b}=$ $10^{-3}$ for $u_{2}(\tau)$ transmission.

\begin{tabular}{lcc}
\hline RS $\left(n^{i}, k^{i}\right)$ & $R_{c}^{i}$ & $t^{i}$ \\
\hline RS $(38,24)$ & 0.63 & 7 \\
RS $(38,28)$ & 0.74 & 5 \\
RS $(38,30)$ & 0.79 & 4 \\
RS $(38,32)$ & 0.84 & 3 \\
RS $(38,34)$ & 0.89 & 2 \\
RS $(38,34)$ & 0.89 & 2 \\
RS $(38,36)$ & 0.95 & 1 \\
RS $(38,36)$ & 0.95 & 1 \\
\hline
\end{tabular}

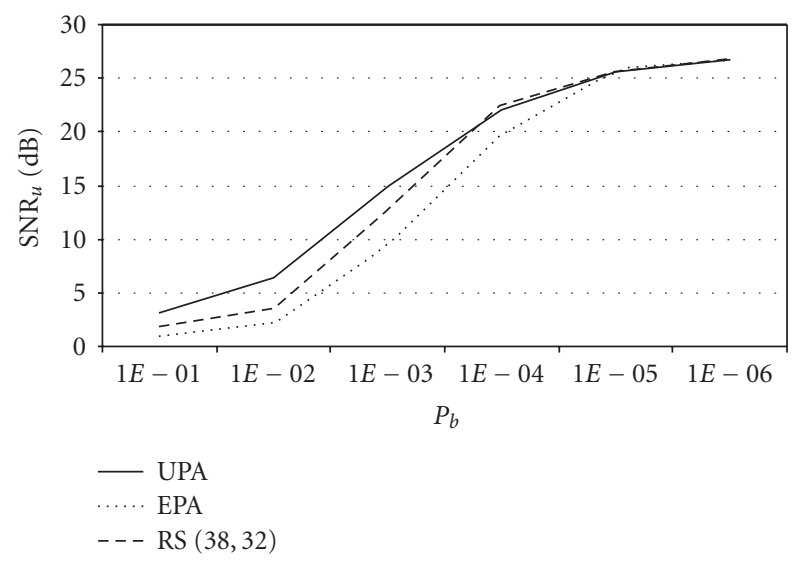

FIGURE 8: Achieved quality for the parameter $u_{1}(\tau)$.

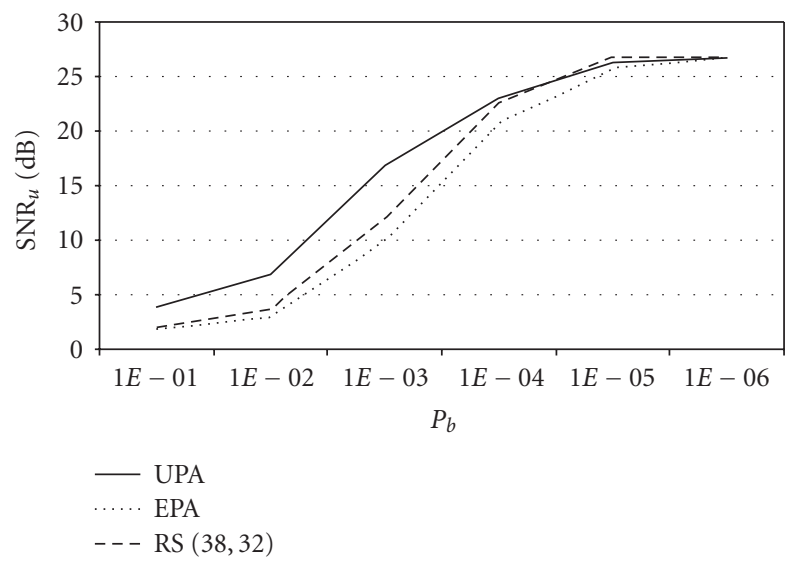

FIGURE 9: Achieved quality for the parameter $u_{2}(\tau)$.

$3.57 \mathrm{~dB}$ the peak gain for $P_{b}=3.5 \times 10^{-3}$ and for $u_{2}(\tau)$. For $P_{b}<10^{-5}$, all the systems perform similarly with slight prevalence of the RS coding which is more evident for $u_{1}(\tau)$. Superior performance in case of $u_{2}(\tau)$ transmission can be justified by the higher precision obtained by a finer power distribution performed with eight weights with respect to a coarser allocation based on only four weights as for $u_{1}(\tau)$. More generally, the UPA prevalence is due to the capability of the optimization procedure to obtain high accuracy by selecting weights in a range of real values.

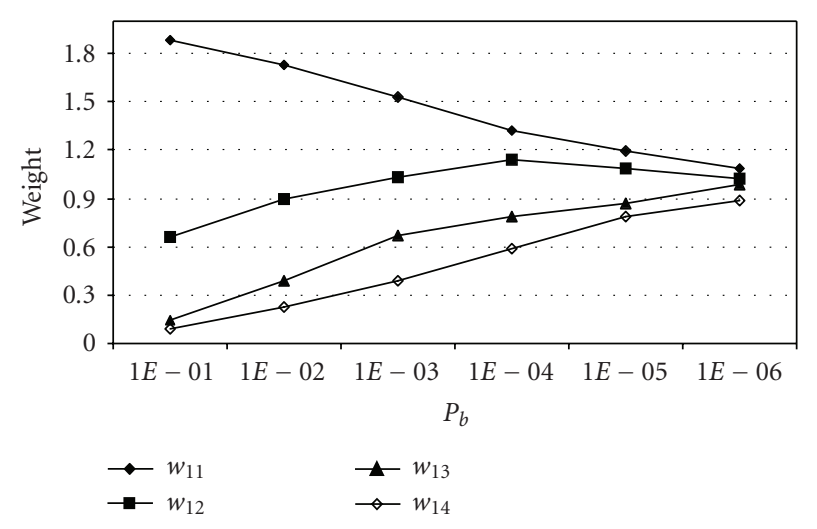

FIGURE 10: Weights values at varying channel conditions for the parameter $u_{1}(\tau)$.

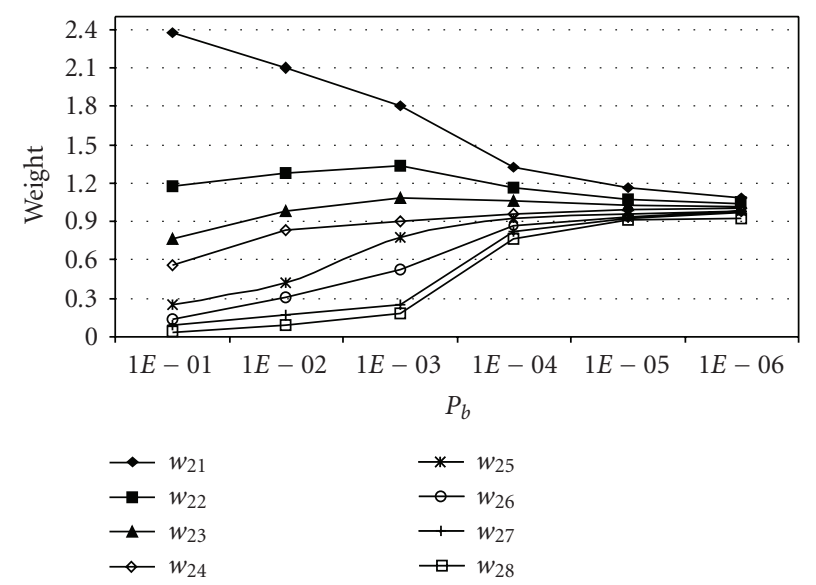

FIGURE 11: Weights values at varying channel conditions for the parameter $u_{2}(\tau)$.

In Figures 10 and 11 show how for severe channel conditions the weights relevant to higher significant bits (i.e., $w_{11}$, $w_{21}$, and $w_{22}$ ) are emphasized with respect to all the others. For $P_{b}$ approaching $10^{-3}$, a decrease of the above weights corresponds to an increase of $w_{12}$ and $w_{23}$ which become also higher than 1. For $P_{b}<10^{-5}$, all the weights converge to equal unitary value, but still remaining slightly different for $P_{b}>10^{-6}$.

Figure 12 shows the percentage bandwidth gain achieved by UPA with respect to UEP based on RS coding for target quality (i.e., fixed $\mathrm{SNR}_{u}[\mathrm{~dB}]$ ) on the transmitted parameters $u_{1}(\tau)$ and $u_{2}(\tau)$, at fixed $P_{b}=10^{-3}$, for the WPDM system used for experiments as represented in Figure 5. A minimum bandwidth gain above $20 \%$ is noticeable whereas similar high variations are observed in both cases. This is due to the discrete nature of RS codes, which are constrained to only a definite set of possible code rates. On the other hand, UPA is a continuous process which guarantees more flexibility in the protection of sensitive data.

In order to assess the suitability of the proposed scheme for real applications, such as audio and video broadcasting, as a further test, we have considered the specific multiplexed 


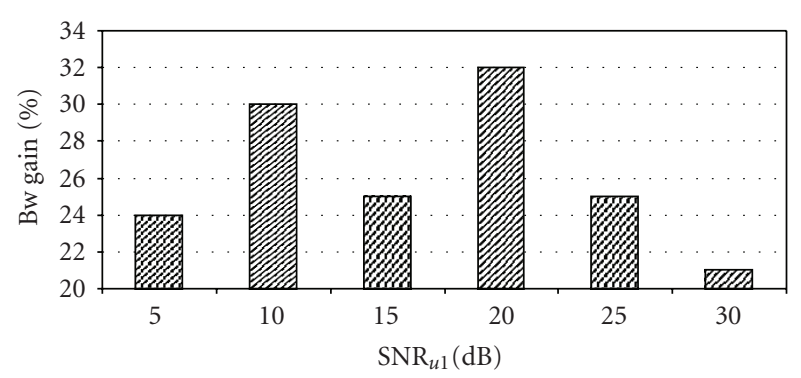

(a)

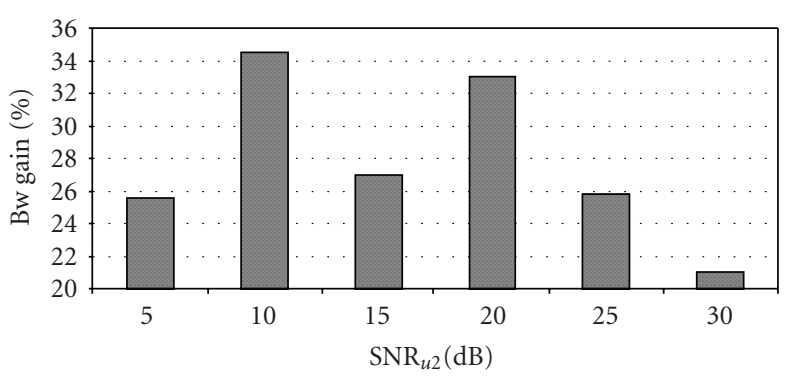

(b)

FIGURE 12: Percentage bandwidth gain for fixed quality on the transmitted parameter $u_{1}(\tau)$ (a) and $u_{2}(\tau)$ (b) achieved by UPA against UEP by RS codes for the WPDM scheme in Figure 7.

TABLE 4: Quality achieved by the proposed system at $P_{b}=10^{-3}$ for the multiple transmission of the image Lena and a stereo-audio CD sequence.

\begin{tabular}{lcc}
\hline Source & PSNR $(\mathrm{dB})$ & PEAQ $(\mathrm{ODG})$ \\
\hline Lena $(512 \times 512, \mathrm{RGB}, 8 \mathrm{bpp})$ & 29.8 & - \\
Stereo-audio CD $(5$ seconds, sampling rate $44.1 \mathrm{KHz}, 16 \mathrm{bps})$ & - & -2.88 \\
\hline
\end{tabular}

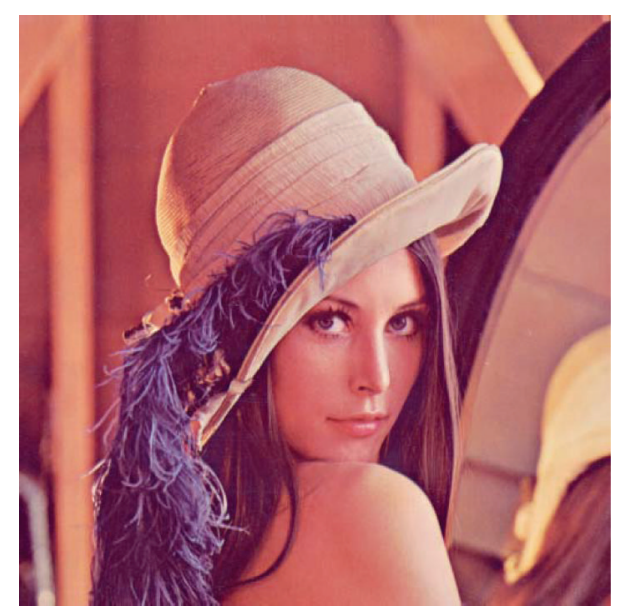

Figure 13: Lena $512 \times 512$ coded in RGB format at 16 bpp per color component.

transmission of a standard image and a stereo-audio sequence. Referring to the system proposed in Figure 7, we have used the well-known image "Lena" of size $512 \times 512$ in RGB format coded at 8 bpp per color component (see Figure 13), as a transmission source $S_{1}$. We have measured the quality on the reconstructed image by standard PSNR metric expressed in decibel. On the other hand, we have ripped a 5 seconds from a stereo-audio CD signal sampled at $44.1 \mathrm{KHz}$ coded at $16 \mathrm{bps}$ and used as a source $S_{2}$. For the evaluation of the quality on the received audio signal, we have used the perceptual evaluation of audio quality (PEAQ) strategy [34]. PEAQ is technique recommended by the ITU, which evaluates the quality of an audio signal by a single number, called objective difference grade (ODG), which varies within a range $[-4 \div 0]$, with 0 the highest quality score. PEAQ has proven to be more performing than conven- tional metrics based on mean square error on the evaluation of the performance of the conventional audio codecs [34].

Table 4 shows the results achieved in case of $P_{b}=10^{-3}$. The quality on the reconstructed image is slightly below $30 \mathrm{~dB}$, whereas the PEAQ measured on the received audio sequence is just up -2.9. This result is in line with the typical performance of low-bit-rate audio and video codecs. For the transmission of audio \video at a rate of $64 \mathrm{Kbit} / \mathrm{s}$, MP3/MPEG-4 codecs achieve PSNR approaching $30 \mathrm{~dB}$ for the reconstructed frames [2] and PEAQ of around -3.36 for the audio sequence [35]. Since conventional DAB and DVB broadcasting systems work at $P_{b} \ll 10^{-3}$, the proposed system could be an alternative solution for the broadcasting of multimedia heterogeneous contents in case of extremely hard transmission condition, when only little quality requirements are set.

\section{CONCLUSION}

In this work, we have presented an orthogonal multiple transmission system based on wavelet packet modulation suitable for the resilient broadcasting of data which demonstrate different sensitivities to transmission errors. A novel unequal error protection technique based on differentiated allocation of the transmission power over the modulated waveforms allows improving the final quality of the received parameters in case of AWGN channel, without any increase of the transmission bandwidth. The optimization of the weights has relied on Genetic Algorithms which allowed to achieve reduced complexity. Due to its scalability properties, the proposed scheme is able to provide for multiple transmissions of heterogeneous services which can be independently protected according to their specific format. Therefore, unequal power allocation applied to wavelet packet division multiplexing offers improved flexibility to broadcaster. Nevertheless, it is worthy to point out that particular attention 
has to be given to the design of the wavelet filters which are real-valued and under the approach of UPA could impair the performance of the transmission in case of wireless systems. In fact, the proposed UPA scheme may increase the dynamic range of the input signals to the WPDM modulator in Figure 4. Since $g_{0}[n]$ is real-causal FIR filter, the bigger input amplitude range may increase the complexity of these filters. This may be a disadvantage of UPA for implementation.

Future work on this subject will investigate the capability of the proposed scheme to deal with real-time varying transmission conditions including the presence of fading effects and the broadcasting of reconfigurable heterogeneous services.

\section{ACKNOWLEDGMENT}

The author would like to thank the three anonymous referees for their constructive comments and suggestions.

\section{REFERENCES}

[1] D. S. Taubman and M. W. Marcellin, JPEG2000: Image Compression Fundamentals, Standards and Practice, Kluwer Academic, Norwell, Mass, USA, 2002.

[2] F. Pereira and T. Ebrahimi, The MPEG-4 Book, IMSC Press Multimedia Series, Prentice-Hall, Englewood Cliffs, NJ, USA, 2002.

[3] K. R. Rao and J. J. Hwang, Techniques \& Standard for Image Video \& Audio Coding, Prentice-Hall, Upper Saddle River, NJ, USA, 1996.

[4] J. G. Proakis, Digital Communications, McGraw-Hill, New York, NY, USA, 4th edition, 2001.

[5] A. Natu and D. Taubman, "Unequal protection of JPEG2000 code-streams in wireless channels," in Proceedings of IEEE Global Telecommunications Conference (GLOBECOM '02), vol. 1, pp. 534-538, Taipei, Taiwan, November 2002.

[6] B. A. Banister, B. Belzer, and T. R. Fischer, "Robust image transmission using JPEG2000 and turbo-codes," IEEE Signal Processing Letters, vol. 9, no. 4, pp. 117-119, 2002.

[7] X. Pan, A. Cuhadar, and A. H. Banihashemi, "Combined source and channel coding with JPEG2000 and ratecompatible low-density parity-check codes," IEEE Transactions on Signal Processing, vol. 54, no. 3, pp. 1160-1164, 2006.

[8] T. Brüggen and P. Vary, "Unequal error protection by modulation with unequal power allocation," IEEE Communications Letters, vol. 9, no. 6, pp. 484-486, 2005.

[9] A. R. Lindsey, "Wavelet packet modulation for orthogonally multiplexed communication," IEEE Transactions on Signal Processing, vol. 45, no. 5, pp. 1336-1339, 1997.

[10] K. M. Wong, J. Wu, T. N. Davidson, and Q. Jin, "Wavelet packet division multiplexing and wavelet packet design under timing error effects," IEEE Transactions on Signal Processing, vol. 45, no. 12, pp. 2877-2890, 1997.

[11] K. M. Wong, J. Wu, T. N. Davidson, Q. Jin, and P.-C. Ching, "Performance of wavelet packet-division multiplexing in impulsive and Gaussian noise," IEEE Transactions on Communications, vol. 48, no. 7, pp. 1083-1086, 2000.

[12] T. N. Davidson, A.-J. Schott, and K. M. Wong, "Branchhopped wavelet packet division multiplexing," in Proceedings of the IEEE International Conference on Acoustics, Speech and Signal Processing (ICASSP '98), vol. 6, pp. 3233-3236, Seattle, Wash, USA, May 1998.
[13] D. Whitley, "A genetic algorithm tutorial," Statistics and Computing, vol. 4, no. 2, pp. 65-85, 1994.

[14] L. Atzori and A. Raccis, "Network capacity assignment for multicast services using genetic algorithms," IEEE Communications Letters, vol. 8, no. 6, pp. 403-405, 2004.

[15] C. W. Ahn and R. S. Ramakrishna, "A genetic algorithm for shortest path routing problem and the sizing of populations," IEEE Transactions on Evolutionary Computation, vol. 6, no. 6, pp. 566-579, 2002.

[16] R. Bhattacharya, P. Venkateswaran, S. K. Sanyal, and R. Nandi, "Genetic algorithm based efficient routing scheme for multicast networks," in Proceedings of IEEE International Conference on Personal Wireless Communications (ICPWC '05), pp. 500504, New Delhi, India, January 2005.

[17] I. Daubechies, Ten Lectures on Wavelets, SIAM, Philadelphia, $\mathrm{Pa}, \mathrm{USA}, 1992$.

[18] A. N. Akansu and R. A. Haddad, Multiresolution Signal Decomposition: Transforms, Subbands, Wavelets, Academic Press, Boston, Mass, USA, 1992.

[19] C. K. Chui, An Introduction to Wavelets, Academic Press, San Diego, Calif, USA, 1992.

[20] P. P. Vaidyanathan, Multirate Systems and Filter Banks, Prentice-Hall, Upper Saddle River, NJ, USA, 1993.

[21] G. G. Walter, Wavelets and Other Orthogonal Systems with Applications, CRC Press, Boca Raton, Fla, USA, 1994.

[22] M. Vetterli and J. Kovacevic, Wavelets and Subband Coding, Prentice-Hall, Englewood Cliffs, NJ, USA, 1995.

[23] G. Strang and T. Nguyen, Wavelets and Filter Banks, WellesleyCambridge Press, Wellesley, Mass, USA, 1996.

[24] G. Strang, "Wavelets and dilation equations: a brief introduction," SIAM Review, vol. 31, no. 4, pp. 614-627, 1989.

[25] O. Rioul and M. Vetterli, "Wavelets and signal processing," IEEE Signal Processing Magazine, vol. 8, no. 4, pp. 14-38, 1991.

[26] B. Jawerth and W. Sweldens, "An overview of wavelet based multiresolution analyses," SIAM Review, vol. 36, no. 3, pp. 377-412, 1994.

[27] A. Cohen and J. Kovacevic, "Wavelets: the mathematical background," Proceedings of the IEEE, vol. 84, no. 4, pp. 514-522, 1996.

[28] N. Hess-Nielsen and M. V. Wickerhauser, "Wavelets and timefrequency analysis," Proceedings of the IEEE, vol. 84, no. 4, pp. 523-540, 1996.

[29] K. Ramchandran, M. Vetterli, and C. Herley, "Wavelets, subband coding, and best bases," Proceedings of the IEEE, vol. 84, no. 4, pp. 541-560, 1996.

[30] S. G. Mallat, "A theory for multiresolution signal decomposition: the wavelet representation," IEEE Transactions on Pattern Analysis and Machine Intelligence, vol. 11, no. 7, pp. 674-693, 1989.

[31] I. Daubechies, "Orthonormal bases of compactly supported wavelets," Communications on Pure and Applied Mathematics, vol. 41, no. 7, pp. 909-996, 1988.

[32] A. Papoulis and S. U. Pillai, Probability, Random Variables, and Stochastic Processes, McGraw-Hill, New York, NY, USA, 2002.

[33] S. Benedetto and E. Biglieri, Principles of Digital Transmission: With Wireless Applications, Kluwer Academic, Norwell, Mass, USA, 1999.

[34] Recommendation ITU-R BS.1387-111/01, "Method for objective measurements of perceived audio quality (PEAQ)".

[35] M. Salovarda, I. Bolkovac, and H. Domitrovic, "Estimating perceptual audio system quality using PEAQ algorithm," in Proceedings of the 18th International Conference on Applied Electromagnetics and Communications (ICECom '05), vol. 1, pp. 1-4, Dubrovnik, Croatia, October 2005. 

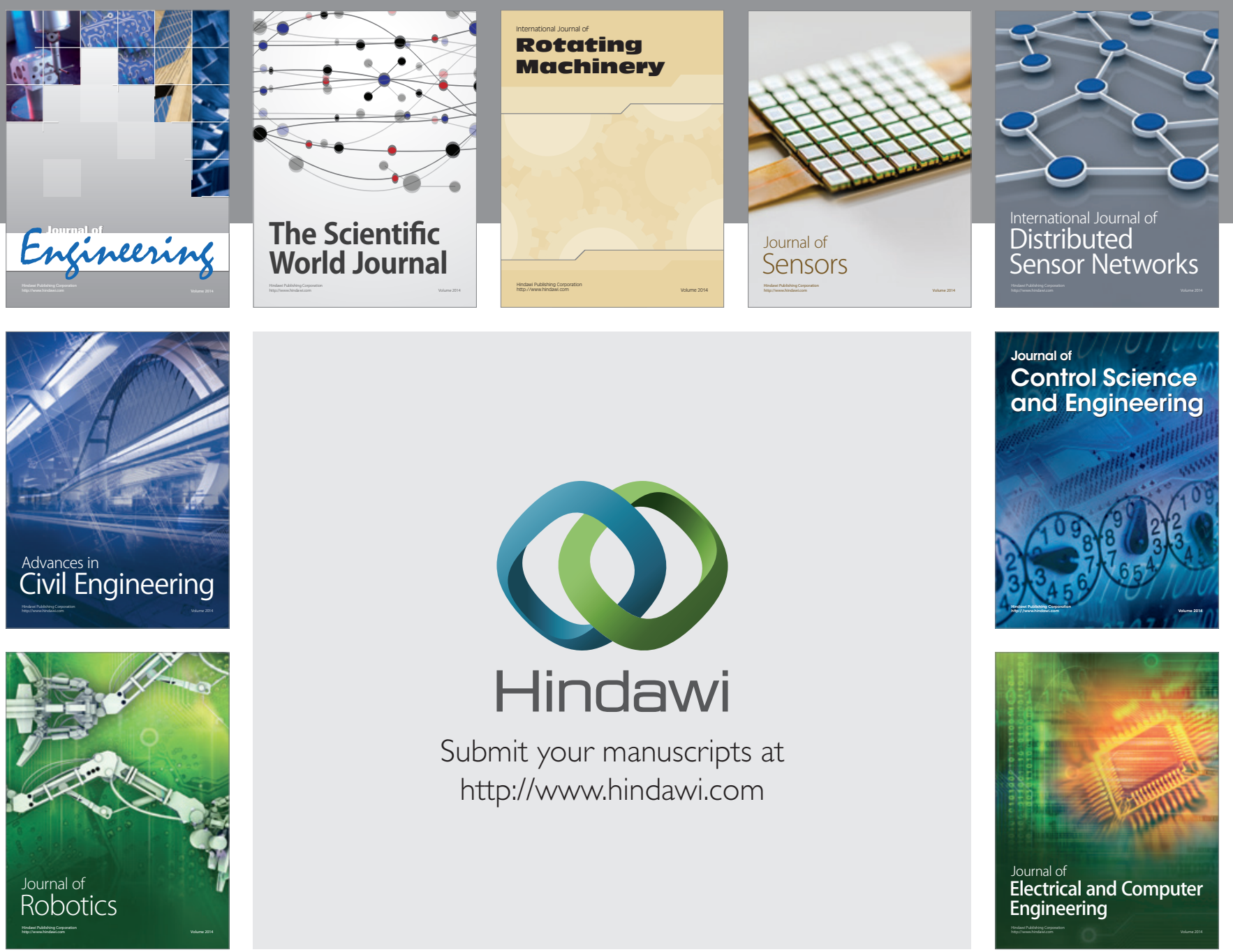

Submit your manuscripts at

http://www.hindawi.com
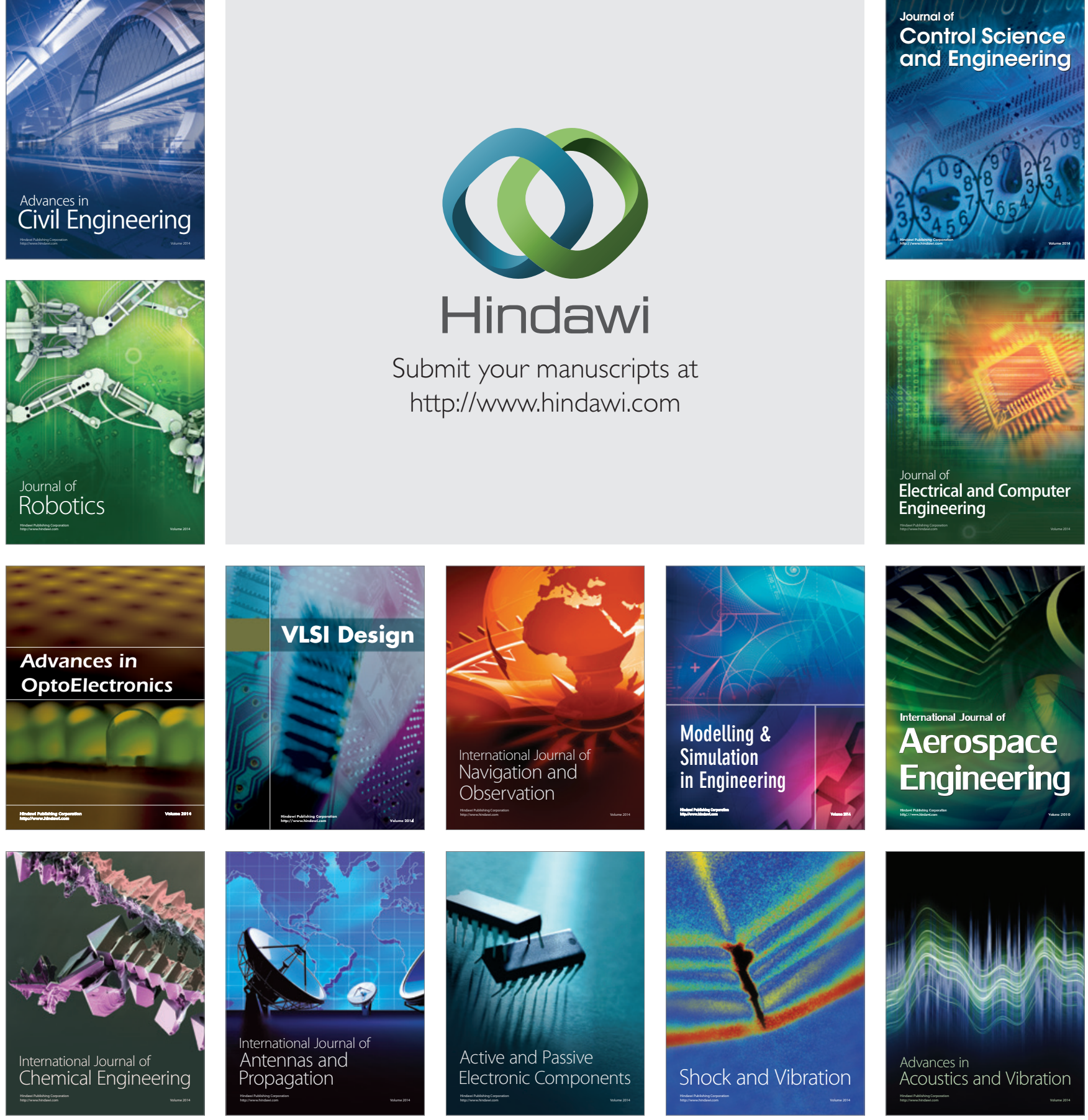